\title{
Cold Hardiness of Primary Buds of Wine and Table Grape Cultivars in Poland
}

\author{
J. Lisek*, A. Lisek \\ Research Institute of Horticulture, Konstytucji 3 Maja 1/3, 96-100 Skierniewice, Poland
}

Submitted for publication: May 2020

Accepted for publication: September 2020

Keywords: Assessment, Central Europe, Vitis spp., winter injuries

\begin{abstract}
The aim of this study was to compare the cold resistance of grapevine cultivars in field conditions. Following the winters of 2016/2017 and 2017/2018, an assessment of frost damage was carried out on the vines of 42 wine cultivars and 45 table grape cultivars grown in central Poland (Skierniewice, latitude 51.9627 N, longitude 20.1666 E). The minimum temperature for each of the two winters was recorded on 2017-01$07\left(-20.9^{\circ} \mathrm{C}\right)$ and on $2018-02-27\left(-20.1^{\circ} \mathrm{C}\right)$. Among the assessed cultivars, 19 (13 wine grape cultivars and six table grape cultivars) belonged to $V$. vinifera species and 68 were inter- or inter-intraspecific hybrids. Cultivars were divided into five classes of different frost tolerance, with information on the proportion of primary buds frozen given in the brackets: very resistant (below $1.9 \%)$, resistant (2\% to $24.9 \%)$, medium susceptible (2\% to $74.9 \%$ ), susceptible $(75 \%$ to $95.9 \%)$ and very susceptible (above $96 \%)$. The number of wine and table grape cultivars in particular classes (mean for two winters) was as follows: very resistant - 20 (wine) and 10 (table), resistant - 15 and 13, medium susceptible - six and 20, susceptible - one and two. Both the $V$. vinifera cultivars and the interspecific hybrids were highly diversified in terms of frost tolerance. In most cultivars, the number of frozen buds after the winter of 2016/2017 was greater than after the winter of 2017/2018. Among $V$. vinifera, 'Riesling', 'Pinot Noir', 'Chasselas Dore' (reference) and 'Tauberschwarz', 'Veltliner Frührot' ('Fruehroter Malvasier'), 'Turan', 'Domina', 'Tressot Panache', 'Blue from Tychy', and 'Irsai Oliver' were very resistant or resistant. Other cultivars of $V$. vinifera were medium susceptible or susceptible.
\end{abstract}

\section{INTRODUCTION}

As a result of climatic changes and progress made in plant breeding, the cultivation of grapevine is being moved north of the traditional areas in Europe and North America (Gustafsson \& Mårtensson, 2005; Rayne et al., 2011). In the past decades a rapid development of vineyards has been observed in Poland and other countries in the cold climatic zone (Vool et al., 2015). This conventional term is used to describe wine-growing regions with a latitude above $50^{\circ} \mathrm{N}$ and cold winters. The frost tolerance of vines is the basic characteristic determining the usefulness for cultivation in areas where winters are cold, such as the central and northern states of the USA, Canada, central and northern Europe (Baltic region), and eastern Europe (Russia and Ukraine). Grapevine genotypes are characterised by varied frost susceptibility, which is related to their origins. Contemporary cultivation takes advantage of inter- and intraspecific hybridisation and develops cultivars that are satisfactorily resistant to frost and produce good-quality fruit (Kostrikin, 1994). American species, such as V. riparia, V. rupestris,
V. lincecumii and $V$. labrusca and the Asian V. amurensis are often used as donors of frost resistance in hybrids with V. vinifera (Alleweldt et al., 1990; Luby, 1991; Kostrikin, 1994; Reisch \& Pratt, 1996; Clark \& Moore, 1999). Frost susceptibility of wintering buds is assessed under laboratory or field conditions (Bourne et al., 1991). Exposure of the scions of one-year-old shoots to low temperature in a cold chamber is a frequently used evaluation method (Cindric \& Korac, 1990). From a practical point of view, field assessment is particularly valuable because it includes the effects of all factors relevant to the level of frost damage. The degree of damage depends not only on minimum temperature and the time of its occurrence, but also on fluctuations in temperature, force of the wind, and the age and physiological state of the plants (Plocher \& Parke, 2001; Fennel, 2004). Current knowledge of the frost susceptibility of particular cultivars is varied. Data on the frost susceptibility of many cultivars is available in scientific papers (Bourne et al., 1991; Clark \& Moore, 1999), textbooks (Pospišilova, 1981; Lott et al.,

\footnotetext{
*Corresponding author: E-mail address: jerzy.lisek@inhort.pl

J. Lisek, ORCID ID: 0000-0002-6374-730X, A. Lisek, ORCID ID: 0000-0002-3421-8759

Acknowledgements: This work was performed in the frame of the multiannual programme on the preservation of gene bank resources, financed by the Polish Ministry of Agriculture and Rural Development: Task 1.3 "Collecting, preservation in ex situ collections, cryoconservation, evaluation, documentation and using gene bank resources of horticultural crops"
} 
2010) and even authorised websites on the Internet (HORT 3040, 2016; Bell, 2018; Vinograd.info, 2019). There is a lack of data on some cultivars recently introduced to Poland from regions with less severe winters. The assessment of frost susceptibility in Poland is being conducted, along with the acquisition of new genotypes (Lisek, 2009, 2012).

The minimum critical (bud-damaging) temperatures tolerated by varieties depend on their ecological-geographical and genetic origin. Among the commonly cultivated varieties of $V$. vinifera, the most resistant to cold are 'Riesling', whose buds tolerate temperature drops to $-21^{\circ} \mathrm{C}$ (Cindric $\&$ Korac, 1990) or even to $-25^{\circ} \mathrm{C}$ (Pospišilova, 1981), and 'Pinot Noir', tolerating temperatures down to $-20^{\circ} \mathrm{C}$ (Pospišilova, 1981). Both varieties come from Western Europe and belong to $V$. vinifera L. subsp. sativa D.C. proles occidentalis Negr. Critical temperatures for cultivars from other ecological-geographical groups of $V$. vinifera, proles pontica and orientalis Negr. are up to $-15^{\circ} \mathrm{C}$ or $-16^{\circ} \mathrm{C}$ (Nikov et al., 1983; Cindric \& Korac, 1990). The frost resistance of interspecific hybrids varies greatly on the basis of their genetic composition and may exceed $-26^{\circ} \mathrm{C}$ (HORT 3040, 2016). Frost damage to buds occurs even when the critical temperature drops for one day (Nikov et al., 1983). In laboratory tests, the critical temperature is maintained for 12 hours (Cindric \& Korac, 1990). The value of critical temperatures changes during the winter, along with physiological changes on a biochemical basis (Ferguson et al., 2014; Nenko et al., 2018). Grapevines acclimate to cold temperatures in the autumn and de-acclimate when warm temperatures return in the spring, and models of bud frost resistance and bud development are variety dependent (Ferguson et al., 2014). In the northern hemisphere, varieties belonging to $V$. vinifera, including subproles occidentalis, achieve maximum frost resistance in the middle of winter (second half of January), hybrids of vinifera $\mathrm{x}$ American species in the middle and at the end of winter (second half of February), and hybrids of vinifera $\mathrm{x}$ amurensis at the beginning (December) and in the middle of winter (Cindric $\&$ Korac, 1990). More rapid de-acclimation and budbreak occur earlier in hardier genotypes originating from colder climates (Ferguson et al., 2014). In areas with cold and long winters at high latitudes, like Poland, damage to the buds occurs most often during deep dormancy (endodormancy), before sap rise, because January and February are the coldest months in the northern hemisphere, including Central Europe (Pospišilova, 1981; data in the present research from Poland). In warmer regions with relatively mild winters and an early spring, critical temperatures may occur at the end of winter, shortly before or after sap rise, and will not be as extremely low as the critical temperatures at the beginning and middle of winter (Ferguson et al., 2014; Nenko et al., 2018).

The aim of this work was to undertake a field assessment of the winter frost susceptibility of wine and table grape cultivars that could possibly be grown in central and northern Europe and that have not yet been methodically assessed in terms of cold resistance. Vines were tested for cultivation in large, commercial vineyards that produce wine, as well as for home garden or agrotourism farm cultivation, without covering the plants for the winter.

\section{MATERIALS AND METHODS}

The assessment of frost damage to the vines was carried out following the winters of 2016/2017 and 2017/2018 in the field collection of the Research Institute of Horticulture in Skierniewice (Poland, latitude $51.9627 \mathrm{~N}$, longitude $20.1666 \mathrm{E})$, located on a luvisol soil, slightly acidic ( $\mathrm{pH}$ 6.3 ) and containing $1.3 \%$ organic matter. Each genotype gathered in the collection was represented by three vines, planted at a spacing of $2.5 \times 1 \mathrm{~m}$ and maintained in the form of a low head with the trunk $0.15 \mathrm{~m}$ to $0.2 \mathrm{~m}$ high and three to five spurs, pruned into two to three buds (six to 10 young shoots per vine). The young shoots were pruned above the $10^{\text {th }}$ to $12^{\text {th }}$ leaf, past the last cluster of grapes, in July. Fertilisation and plant protection were carried out according to current recommendations for commercial vineyards. Mineral fertilisation - 'Azofoska', a multicomponent fertiliser containing macronutrients $\mathrm{N}, \mathrm{P}, \mathrm{K}$ and $\mathrm{Mg}$ and micronutrients $\mathrm{Cu}, \mathrm{Zn}, \mathrm{Mn}, \mathrm{B}$ and $\mathrm{Mo}$, and triple superphosphate $(\mathrm{P})$ were applied in the spring, at the beginning of April. Half of the necessary nitrogen dose was applied in the first half of June in the form of calcium nitrate. The annual dose of macronutrients was $\mathrm{N}: 40 \mathrm{~kg}$ $\mathrm{ha}^{-1}$, P: $30 \mathrm{~kg} \mathrm{ha}^{-1}, \mathrm{~K}: 100 \mathrm{~kg} \mathrm{ha}^{-1}$. A chemical protection against fungal diseases was carried out, using fungicides containing copper, sulphur (two treatments per season by each agent), mancozeb+metalaxyl, pyraclostrobin+boscalid, cyprodynil+fludioxonil, penconazole (optionally, one to two treatments per season by product, depending on the weather). Vines were covered with cereal straw (mounds of $0.4 \mathrm{~m}$ ) for the winter (December).

Frost damage to the vines was assessed after the winters, which were characterised by weather conditions as set out in the Table 1. The sum of active temperatures (SAT) - medium daily temperature from April to October, higher than $10^{\circ} \mathrm{C}-$ amounted to $2667^{\circ} \mathrm{C}$ (growing degree days, GDD - 1 098) in 2016 , and $2693^{\circ} \mathrm{C}$ (GDD - 1 030) in 2017, which did not diverge from the average of the preceding ten years. There were no drops in temperature below $-10^{\circ} \mathrm{C}$ in November 2016 and 2017, which allowed the plants to become adapted to winter conditions gradually. Extremely low temperatures occurred in the first two weeks of January 2017 (winter 2016/2017), and in last week of February and the first days of March 2018 (winter 2017/2018). The minimum temperature at a height of 2.0 metres recorded on 2017-0107 was $-20.9^{\circ} \mathrm{C}$. At a height of 0.2 metres the temperature dropped to $-22.3^{\circ} \mathrm{C}$. The minimum temperature of the next winter was $-20.1^{\circ} \mathrm{C}\left(-20.6^{\circ} \mathrm{C}\right.$ at a height of 0.2 metres $)$ and was recorded on 2018-02-27.

Forty-two grapevine cultivars valuable for wine production and 45 grapevine cultivars for table grapes (including reference cultivars) were selected for the study out of the 320 genotypes represented in the collection. From among the wine cultivars, the following were selected as references: 'Marechal Foch' (interspecific hybrid), 'Solaris' and 'Regent' (inter-intraspecific hybrids), 'Riesling' and 'Pinot Noir' ( $V$. vinifera). Inter-intraspecific (intrainterspecific) hybrids were isolated into a separate group (Theocharis et al., 2010), and classified as Vitis vinifera cultivars (Vitis International Variety Catalogue, 2019). 'Muscat Bleu' and 'Chasselas Dore' were chosen as reference 
TABLE 1

Climatic conditions in Skierniewice in the period July 2016 to March 2018

\begin{tabular}{|c|c|c|c|c|c|}
\hline \multirow[b]{2}{*}{ Year } & \multirow[b]{2}{*}{ Month } & \multicolumn{2}{|c|}{$\begin{array}{l}\text { Air temperature at the height of } 2.0 \mathrm{~m} \\
\text { and date on which it occurred }\left({ }^{\circ} \mathrm{C}\right)\end{array}$} & \multirow{2}{*}{$\begin{array}{l}\text { Average monthly air } \\
\text { temperature }\left({ }^{\circ} \mathrm{C}\right)\end{array}$} & \multirow{2}{*}{$\begin{array}{l}\text { Precipitation } \\
\quad(\mathrm{mm})\end{array}$} \\
\hline & & Minimum & Maximum & & \\
\hline \multirow[t]{6}{*}{2016} & July & $7.2(22.07)$ & $32.0(11.07)$ & 18.7 & 103.8 \\
\hline & August & $5.5(12.08)$ & $30.7(05.08)$ & 17.9 & 0.2 \\
\hline & September & $-2.0(27.09)$ & $30.0(12.09)$ & 14.9 & 0.8 \\
\hline & October & $-1.8(07.10)$ & $21.5(02.10)$ & 7.1 & 32.6 \\
\hline & November & $-6.2(10.11)$ & $14.2(21.11)$ & 3.0 & 0.6 \\
\hline & December & $-8.1(12.12)$ & $9.4(26.12)$ & 1.2 & 1.6 \\
\hline \multirow[t]{12}{*}{2017} & January & $-20.9(07.01)$ & $3.8(13.01)$ & -4.3 & 9.0 \\
\hline & February & $-12.2(13.02)$ & $13.2(27.02)$ & -1.2 & 34.8 \\
\hline & March & $-1.5(08.03)$ & $20.5(31.03)$ & 5.9 & 46.8 \\
\hline & April & $-4.0(17.04)$ & $22.6(02.04)$ & 7.0 & 72.2 \\
\hline & May & $-2.5(10.05)$ & $27.6(20.05)$ & 13.6 & 54.4 \\
\hline & June & $7.2(02.06)$ & $29.5(20.06)$ & 17.5 & 149.8 \\
\hline & July & $6.5(06.07)$ & $32.0(30.07)$ & 18.2 & 51.8 \\
\hline & August & $6.4(29.08)$ & $35.0(01.08)$ & 18.9 & 71.8 \\
\hline & September & $2.1(29.09)$ & $23.5(10.09)$ & 13.3 & 249.4 \\
\hline & October & $0.6(31.10)$ & $21.3(16.10)$ & 9.6 & 77.6 \\
\hline & November & $-1.5(22.11)$ & $12.4(05.11)$ & 4.7 & 41.4 \\
\hline & December & $-3.5(20.12)$ & $10.9(12.12)$ & 2.3 & 67.6 \\
\hline \multirow[t]{3}{*}{2018} & January & $-8.6(16.01)$ & $9.9(29.01)$ & 0.6 & 22.2 \\
\hline & February & $-20.1(27.02)$ & $5.5(01.02)$ & -3.6 & 8.0 \\
\hline & March & $-19.4(02.03)$ & $11.7(30.03)$ & 0.2 & 15.8 \\
\hline
\end{tabular}

table grape cultivars. Vines of the assessed cultivars were planted in 2012. Information on wine and table grape cultivars, such as origin and skin colour of the berries, is given in Tables 2 and 3, which also contain the results of the study. Frost damage to the buds was assessed at the time of cutting back the woody shoots at the end of March and at the beginning of April. Three samples of cane were taken from each of the plots (cultivars), each with 50 buds from the three plants being assessed. One-year-old completely woody shoots of the evaluated cultivars, with tested primary buds free from mechanical damage and symptoms of disease, were taken from the parts of vines that were not covered with straw or snow during the winter, at a height of $0.5 \mathrm{~m}$ to $1.5 \mathrm{~m}$ above the surface of the ground. The buds under assessment were cut across, and the colour of the incipient shoots was determined. An overwintering bud was considered to be dead if both the central bud and the lateral buds underneath were dark brown. The obtained results, expressed as a percentage of the buds damaged by frost in relation to the total number of buds, were analysed statistically using the variance analysis method, which was applied to the values after Bliss's transformation. The significance of the means was evaluated using Duncan's test at the 5\% level. Cultivars were divided into five classes of different frost tolerance according to the percentage of frozen buds: very resistant (below $1.9 \%$ ), resistant ( $2 \%$ to $24.9 \%$ ), medium susceptible ( $25 \%$ to $74.9 \%)$, susceptible $(75 \%$ to $95.9 \%)$ and very susceptible (above 96\%).

\section{RESULTS AND DISCUSSION}

For most cultivars, frost injuries to primary buds were greater after the winter of 2016/2017 than after the winter of 2017/2018 (Tables 2 and 3). During the assessment in the spring of 2017, one wine cultivar, 'Cabernet Mitos', and three table grape cultivars, 'Vienetchnij', 'Philipp' and 'Evita', were included in the very susceptible class. After expanding the results as a mean of a two-winter assessment, the number of wine and table grape cultivars in particular classes was as follows: very resistant -20 (wine) and 10 (table), resistant - 15 and 13, medium susceptible - six and 20, and susceptible - one and two.

Interspecific hybrids originating from the US - 'E.S. N-5-10', 'Frontenac', 'Kay Gray', 'Marquette', 'Norway Red', 'Sabrevois', 'St. Croix', 'St. Pepin' and 'Troubadour'; from the Ukraine - 'Muskat Odesskij'; and from Canada - 'Vandal Cliché', proved to be the wine cultivars most tolerant to cold damage. During the study, wintering buds of those cultivars were not damaged by frost - the same as in the case of the reference hybrid, 'Marechal Foch', which is widely grown in countries with cold winters (Plocher \& Parke, 2001).

Cultivars classified as inter-intraspecific hybrids bred in Germany were characterised by varied frost tolerance. 'Baron' and 'Hibernal' were the most resistant. Among cultivars most often grown in the Baltic region, including Poland, and taking into consideration the mean results of two winters, 'Solaris' was very resistant and 'Regent' was resistant. Some of the inter-intraspecific hybrids, including 'Regent' and 'Calandro', were far inferior in frost resistance 
TABLE 2

Cold damage to wine grapevines following the winters of 2016/2017 and 2017/2018, Skierniewice.

\begin{tabular}{|c|c|c|c|c|c|c|c|}
\hline \multirow[b]{2}{*}{ Cultivar } & \multirow{2}{*}{$\begin{array}{l}\text { Colour of } \\
\text { berry skin* }\end{array}$} & \multirow[b]{2}{*}{ Origin** } & \multirow{2}{*}{$\begin{array}{c}\text { Country of } \\
\text { breeding }\end{array}$} & \multicolumn{3}{|c|}{ Frozen buds $(\%)$} & \multirow{2}{*}{$\begin{array}{l}\text { Class of frost } \\
\text { tolerance*** } \\
\text { (mean) }\end{array}$} \\
\hline & & & & $2016 / 2017$ & $2017 / 2018$ & Mean & \\
\hline Baron & $\mathrm{N}$ & IIH & Germany & $0.0 \mathrm{a}$ & $0.0 \mathrm{a}$ & $0.0 \mathrm{a}$ & VR \\
\hline E.S. N-5-10 & $\mathrm{R}-\mathrm{N}$ & $\mathrm{IH}$ & USA & $0.0 \mathrm{a}$ & $0.0 \mathrm{a}$ & $0.0 \mathrm{a}$ & VR \\
\hline Frontenac & $\mathrm{N}$ & $\mathrm{IH}$ & USA & $0.0 \mathrm{a}$ & $0.0 \mathrm{a}$ & $0.0 \mathrm{a}$ & VR \\
\hline Kay Gray & $\mathrm{B}$ & $\mathrm{IH}$ & USA & $0.0 \mathrm{a}$ & $0.0 \mathrm{a}$ & $0.0 \mathrm{a}$ & VR \\
\hline Marechal Foch & $\mathrm{N}$ & $\mathrm{IH}$ & France & $0.0 \mathrm{a}$ & $0.0 \mathrm{a}$ & $0.0 \mathrm{a}$ & VR \\
\hline Marquette & $\mathrm{N}$ & $\mathrm{IH}$ & USA & $0.0 \mathrm{a}$ & $0.0 \mathrm{a}$ & $0.0 \mathrm{a}$ & VR \\
\hline Muskat Odesskij & $\mathrm{B}$ & $\mathrm{IH}$ & Ukraine & $0.0 \mathrm{a}$ & $0.0 \mathrm{a}$ & $0.0 \mathrm{a}$ & VR \\
\hline Norway Red & $\mathrm{N}$ & $\mathrm{IH}$ & USA & $0.0 \mathrm{a}$ & $0.0 \mathrm{a}$ & $0.0 \mathrm{a}$ & VR \\
\hline Sabrevois & $\mathrm{N}$ & $\mathrm{IH}$ & USA & $0.0 \mathrm{a}$ & $0.0 \mathrm{a}$ & $0.0 \mathrm{a}$ & VR \\
\hline St. Croix & $\mathrm{N}$ & $\mathrm{IH}$ & USA & $0.0 \mathrm{a}$ & $0.0 \mathrm{a}$ & $0.0 \mathrm{a}$ & VR \\
\hline St. Pepin & $\mathrm{B}$ & $\mathrm{IH}$ & USA & $0.0 \mathrm{a}$ & $0.0 \mathrm{a}$ & $0.0 \mathrm{a}$ & VR \\
\hline Troubadour & $\mathrm{N}$ & $\mathrm{IH}$ & USA & $0.0 \mathrm{a}$ & $0.0 \mathrm{a}$ & $0.0 \mathrm{a}$ & VR \\
\hline Vandal Cliché & $\mathrm{B}$ & $\mathrm{IH}$ & Canada & $0.0 \mathrm{a}$ & $0.0 \mathrm{a}$ & $0.0 \mathrm{a}$ & VR \\
\hline Hibernal & $\mathrm{B}$ & $\mathrm{IIH}$ & Germany & $0.2 \mathrm{ab}$ & $0.0 \mathrm{a}$ & $0.1 \mathrm{ab}$ & VR \\
\hline Allegro & $\mathrm{N}$ & IIH & Germany & $0.9 \mathrm{bc}$ & $0.0 \mathrm{a}$ & $0.2 \mathrm{a}-\mathrm{c}$ & VR \\
\hline Helios & $\mathrm{B}$ & IIH & Germany & $2.0 \mathrm{~cd}$ & $0.0 \mathrm{a}$ & $0.5 \mathrm{a}-\mathrm{c}$ & VR \\
\hline Jutrzenka & $\mathrm{B}$ & $\mathrm{IH}$ & Poland & $2.0 \mathrm{~cd}$ & $0.0 \mathrm{a}$ & $0.5 \mathrm{a}-\mathrm{c}$ & VR \\
\hline Riesling & $\mathrm{B}$ & $\operatorname{Vin}$ & Germany & $2,6 \mathrm{de}$ & $0.0 \mathrm{a}$ & $0.7 \mathrm{a}-\mathrm{c}$ & VR \\
\hline Souvignier Gris & $\mathrm{R}$ & $\mathrm{IIH}$ & Germany & $4.0 \mathrm{ef}$ & $0.0 \mathrm{a}$ & $1.0 \mathrm{a}-\mathrm{c}$ & VR \\
\hline Solaris & $\mathrm{B}$ & IIH & Germany & $6.6 \mathrm{gh}$ & $0.0 \mathrm{a}$ & $1.7 \mathrm{a}-\mathrm{c}$ & VR \\
\hline Felicia & $\mathrm{B}$ & IIH & Germany & $9.3 \mathrm{hi}$ & $0.0 \mathrm{a}$ & $2.4 \mathrm{a}-\mathrm{c}$ & $\mathrm{R}$ \\
\hline Bolero & $\mathrm{N}$ & IIH & Germany & $10.6 \mathrm{i}$ & $0.0 \mathrm{a}$ & $2.7 \mathrm{~b}-\mathrm{d}$ & $\mathrm{R}$ \\
\hline Hybrid from Karpicko & $\mathrm{N}$ & $\mathrm{IH}$ & Poland & $12.0 \mathrm{i}$ & $0.0 \mathrm{a}$ & $3.1 \mathrm{~b}-\mathrm{e}$ & $\mathrm{R}$ \\
\hline Villaris & $\mathrm{B}$ & $\mathrm{IIH}$ & Germany & $12.0 \mathrm{i}$ & $0.0 \mathrm{a}$ & $3.1 \mathrm{~b}-\mathrm{e}$ & $\mathrm{R}$ \\
\hline Cabernet Cantor & $\mathrm{N}$ & IIH & Germany & $9.3 \mathrm{hi}$ & $0.2 \mathrm{~b}$ & $3.2 \mathrm{~b}-\mathrm{e}$ & $\mathrm{R}$ \\
\hline Pinot Noir & $\mathrm{N}$ & $\operatorname{Vin}$ & France & $8.6 \mathrm{hi}$ & $0.9 \mathrm{c}$ & $3.8 \mathrm{c}-\mathrm{e}$ & $\mathrm{R}$ \\
\hline Tauberschwarz & $\mathrm{N}$ & $\operatorname{Vin}$ & Germany & $5.3 \mathrm{fg}$ & $3.3 \mathrm{~d}$ & $4.2 \mathrm{c}-\mathrm{e}$ & $\mathrm{R}$ \\
\hline Veltliner Frührot & $\mathrm{R}$ & $\operatorname{Vin}$ & Austria & $26.0 \mathrm{j}$ & $0.9 \mathrm{c}$ & $9.6 \mathrm{~d}-\mathrm{f}$ & $\mathrm{R}$ \\
\hline Muscaris & $\mathrm{B}$ & IIH & Germany & $35.3 \mathrm{mn}$ & $0.0 \mathrm{a}$ & $9.8 \mathrm{~d}-\mathrm{f}$ & $\mathrm{R}$ \\
\hline Monarch & $\mathrm{N}$ & IIH & Germany & $36.0 \mathrm{mn}$ & $0.2 \mathrm{~b}$ & 10.0 ef & $\mathrm{R}$ \\
\hline Calandro & $\mathrm{N}$ & IIH & Germany & $31.3 \mathrm{k}-\mathrm{m}$ & $6.6 \mathrm{e}$ & $17.2 \mathrm{fg}$ & $\mathrm{R}$ \\
\hline Sirius & $\mathrm{B}$ & IIH & Germany & $40.7 \mathrm{n}$ & $6.6 \mathrm{e}$ & $21.5 \mathrm{~g}$ & $\mathrm{R}$ \\
\hline Turan & $\mathrm{N}$ & Vin & Hungary & $29.3 \mathrm{j}-1$ & $14.7 \mathrm{~g}$ & $21.5 \mathrm{~g}$ & $\mathrm{R}$ \\
\hline Regent & $\mathrm{N}$ & IIH & Germany & $30.6 \mathrm{j}-\mathrm{m}$ & $9.3 \mathrm{f}$ & $18.7 \mathrm{fg}$ & $\mathrm{R}$ \\
\hline Domina & $\mathrm{N}$ & $\operatorname{Vin}$ & Germany & $26.7 \mathrm{jk}$ & $18.0 \mathrm{~g}$ & $22.5 \mathrm{~g}$ & $\mathrm{R}$ \\
\hline Bouvier & $\mathrm{B}$ & $\operatorname{Vin}$ & Slovenia & $35.3 \mathrm{mn}$ & $16.7 \mathrm{~g}$ & $25.4 \mathrm{~g}$ & MS \\
\hline Merlot & $\mathrm{N}$ & $\operatorname{Vin}$ & France & $32.6 \mathrm{~lm}$ & $23.3 \mathrm{~h}$ & $27.9 \mathrm{~g}$ & MS \\
\hline Schönburger & $\mathrm{R}$ & Vin & Germany & $68.0 \mathrm{p}$ & $16.7 \mathrm{~g}$ & $41.0 \mathrm{~h}$ & MS \\
\hline Morio Muscat & $\mathrm{B}$ & $\operatorname{Vin}$ & Germany & 65.4 op & $25.3 \mathrm{~h}$ & $44.9 \mathrm{~h}$ & MS \\
\hline Syrah & $\mathrm{N}$ & $\operatorname{Vin}$ & France & $62.7 \mathrm{o}$ & $29.3 \mathrm{i}$ & $45.4 \mathrm{jk}$ & MS \\
\hline Dunaj & $\mathrm{N}$ & $\operatorname{Vin}$ & Slovakia & $90.7 \mathrm{q}$ & $24.0 \mathrm{~h}$ & $60.0 \mathrm{i}$ & MS \\
\hline Cabernet Mitos & $\mathrm{N}$ & $\operatorname{Vin}$ & Germany & $99.8 \mathrm{r}$ & $76.1 \mathrm{j}$ & $92.4 \mathrm{j}$ & $\mathrm{S}$ \\
\hline
\end{tabular}

Means followed by the same letter do not differ significantly at $\mathrm{p}=0.05$.

* Colour of berry skin: B (blanc) - green-yellow; R - rose; N (noir) - blue-black

** Origin: Vin - V. vinifera; IH - interspecific hybrid from crossing V. vinifera and species from among V. riparia, V. rupestris, V. lincecumii, $V$. amurensis and $V$. labrusca; $\mathrm{IIH}$ - cultivar from crossing interspecific hybrid and $V$. vinifera

*** Class of frost tolerance, with percentage of frozen buds in brackets: VR - very resistant (below 1.9\%), R - resistant (2\% to $24.9 \%$ ), MS

- medium susceptible ( $25 \%$ to $74.9 \%$ ), S - susceptible ( $75 \%$ to $95.9 \%$ ) and VS - very susceptible (above $96 \%$ ). 
TABLE 3

Cold damage to table grapevines following the winters of 2016/2017 and 2017/2018, Skierniewice.

\begin{tabular}{|c|c|c|c|c|c|c|c|}
\hline \multirow[b]{2}{*}{ Cultivar } & \multirow{2}{*}{$\begin{array}{l}\text { Colour of } \\
\text { berry skin* }\end{array}$} & \multirow[b]{2}{*}{ Origin** } & \multirow{2}{*}{$\begin{array}{c}\text { Country of } \\
\text { breeding }\end{array}$} & \multicolumn{3}{|c|}{ Frozen buds $(\%)$} & \multirow{2}{*}{$\begin{array}{l}\text { Class of frost } \\
\text { tolerance*** } \\
\quad(\text { mean })\end{array}$} \\
\hline & & & & $2016 / 2017$ & $2017 / 2018$ & Mean & \\
\hline Bluebell & $\mathrm{N}$ & $\mathrm{IH}$ & USA & $0.0 \mathrm{a}$ & $0.0 \mathrm{a}$ & $0.0 \mathrm{a}$ & VR \\
\hline Izopan & $\mathrm{B}$ & $\mathrm{IH}$ & Poland & $0.0 \mathrm{a}$ & $0.0 \mathrm{a}$ & $0.0 \mathrm{a}$ & VR \\
\hline Kivikrass & $\mathrm{N}$ & $\mathrm{IH}$ & Estonia & $0.0 \mathrm{a}$ & $0.0 \mathrm{a}$ & $0.0 \mathrm{a}$ & VR \\
\hline Mars Sdl (seedless) & $\mathrm{N}$ & $\mathrm{IH}$ & USA & $0.0 \mathrm{a}$ & $0.0 \mathrm{a}$ & $0.0 \mathrm{a}$ & VR \\
\hline Siewiernij Rannij & $\mathrm{B}$ & $\mathrm{IH}$ & Russia & $0.0 \mathrm{a}$ & $0.0 \mathrm{a}$ & $0.0 \mathrm{a}$ & VR \\
\hline Somerset Sdl & $\mathrm{R}$ & $\mathrm{IH}$ & USA & $0.0 \mathrm{a}$ & $0.0 \mathrm{a}$ & $0.0 \mathrm{a}$ & VR \\
\hline Trollhaugen Sdl & $\mathrm{N}$ & $\mathrm{IH}$ & USA & $0.0 \mathrm{a}$ & $0.0 \mathrm{a}$ & $0.0 \mathrm{a}$ & VR \\
\hline Vanessa Sdl & $\mathrm{R}$ & $\mathrm{IH}$ & Canada & $0.0 \mathrm{a}$ & $0.0 \mathrm{a}$ & $0.0 \mathrm{a}$ & VR \\
\hline Venus Sdl & $\mathrm{N}$ & $\mathrm{IH}$ & USA & $0.0 \mathrm{a}$ & $0.0 \mathrm{a}$ & $0.0 \mathrm{a}$ & VR \\
\hline Jupiter Sdl & $\mathrm{R}-\mathrm{N}$ & $\mathrm{IH}$ & USA & $0.2 \mathrm{a}$ & $0.2 \mathrm{~b}$ & $0.2 \mathrm{ab}$ & VR \\
\hline Muscat Bleu & $\mathrm{N}$ & $\mathrm{IH}$ & Switzerland & $8.6 \mathrm{~b}$ & $0.0 \mathrm{a}$ & $2.2 \mathrm{a}-\mathrm{c}$ & $\mathrm{R}$ \\
\hline Tressot Panache & $\mathrm{N}(\mathrm{B})$ & $\operatorname{Vin}$ & France & $5.3 \mathrm{~b}$ & $2.0 \mathrm{c}$ & $3.5 \mathrm{bc}$ & $\mathrm{R}$ \\
\hline Marquis Sdl & B & $\mathrm{IH}$ & USA & $5.3 \mathrm{~b}$ & $3.3 \mathrm{~cd}$ & $4.2 \mathrm{~b}-\mathrm{d}$ & $\mathrm{R}$ \\
\hline Zodiak & $\mathrm{R}$ & $\mathrm{IH}$ & Russia & $6.0 \mathrm{~b}$ & $4.6 \mathrm{~d}$ & $5.3 \mathrm{~b}-\mathrm{e}$ & $\mathrm{R}$ \\
\hline Zoltaja Lora & $\mathrm{B}$ & $\mathrm{IH}$ & Ukraine & $14.6 \mathrm{c}$ & $4.6 \mathrm{~d}$ & $9.0 \mathrm{c}-\mathrm{f}$ & $\mathrm{R}$ \\
\hline Chasselas Dore & $\mathrm{B}$ & Vin & Unknown & $22.0 \mathrm{~d}$ & $7.2 \mathrm{e}$ & $13.8 \mathrm{~d}-\mathrm{g}$ & $\mathrm{R}$ \\
\hline Pleven Muskatnyj & $\mathrm{B}$ & $\mathrm{IH}$ & Bulgaria & $22.0 \mathrm{~d}$ & $8.6 \mathrm{ef}$ & $14.7 \mathrm{~d}-\mathrm{h}$ & $\mathrm{R}$ \\
\hline Blue from Tychy & $\mathrm{N}$ & Vin & Unknown & $22.0 \mathrm{~d}$ & $9.3 \mathrm{e}-\mathrm{g}$ & $15.1 \mathrm{e}-\mathrm{h}$ & $\mathrm{R}$ \\
\hline Charlie (Antracit) & $\mathrm{N}$ & $\mathrm{IH}$ & Russia & $32.5 \mathrm{ef}$ & $7.9 \mathrm{e}$ & $18.6 \mathrm{f}-\mathrm{i}$ & $\mathrm{R}$ \\
\hline Sophie & $\mathrm{B}$ & $\mathrm{IH}$ & Hungary & $30.6 \mathrm{e}$ & $9.3 \mathrm{e}-\mathrm{g}$ & $18.8 \mathrm{f}-\mathrm{i}$ & $\mathrm{R}$ \\
\hline Irsai Oliver & B & Vin & Hungary & $22.6 \mathrm{~d}$ & $18.8 \mathrm{~m}$ & $20.6 \mathrm{f}-\mathrm{j}$ & $\mathrm{R}$ \\
\hline Krasotka & $\mathrm{R}$ & $\mathrm{IH}$ & Russia & $38.0 \mathrm{f}$ & $8.6 \mathrm{ef}$ & $21.4 \mathrm{f}-\mathrm{j}$ & $\mathrm{R}$ \\
\hline Piesnia & $\mathrm{B}$ & $\mathrm{IH}$ & Russia & $28.5 \mathrm{e}$ & $18.0 \mathrm{~lm}$ & $23.0 \mathrm{f}-\mathrm{k}$ & $\mathrm{R}$ \\
\hline Aron & $\mathrm{B}$ & $\mathrm{IH}$ & Hungary & $45.3 \mathrm{~g}$ & $11.3 \mathrm{f}-\mathrm{h}$ & $26.5 \mathrm{~g}-1$ & MS \\
\hline Romulus Sdl & $\mathrm{B}$ & $\mathrm{IH}$ & USA & $45.3 \mathrm{~g}$ & $14.6 \mathrm{i}-1$ & $28.7 \mathrm{~g}-\mathrm{m}$ & MS \\
\hline Oryginal Tchernij & $\mathrm{N}$ & $\mathrm{IH}$ & Ukraine & $55.3 \mathrm{hi}$ & $11.3 \mathrm{f}-\mathrm{h}$ & $31.0 \mathrm{~h}-\mathrm{m}$ & MS \\
\hline Laura & $\mathrm{R}$ & $\mathrm{IH}$ & Ukraine & $60.7 \mathrm{ij}$ & $12.7 \mathrm{~h}-\mathrm{j}$ & $34.5 \mathrm{i}-\mathrm{n}$ & MS \\
\hline Saturn Sdl & $\mathrm{R}-\mathrm{N}$ & $\mathrm{IH}$ & USA & $62.0 \mathrm{ij}$ & $14.7 \mathrm{i}-1$ & $36.6 \mathrm{j}-\mathrm{n}$ & MS \\
\hline Stefanie & B & $\mathrm{IH}$ & Austria & $65.4 \mathrm{j}$ & $13.3 \mathrm{~h}-\mathrm{k}$ & $37.4 \mathrm{j}-\mathrm{n}$ & MS \\
\hline White from Leszno & $\mathrm{B}$ & $\operatorname{Vin}$ & Poland & $51.3 \mathrm{gh}$ & $26.3 \mathrm{n}$ & $38.6 \mathrm{j}-\mathrm{n}$ & MS \\
\hline Chryzolit & $\mathrm{B}$ & $\mathrm{IH}$ & Russia & $66.0 \mathrm{j}$ & $17.3 \mathrm{~lm}$ & $40.4 \mathrm{k}-\mathrm{n}$ & MS \\
\hline Katharina & $\mathrm{R}$ & $\mathrm{IH}$ & Austria & $50.7 \mathrm{gh}$ & $31.3 \mathrm{o}$ & $40.8 \mathrm{k}-\mathrm{n}$ & MS \\
\hline Argo & $\mathrm{R}$ & $\mathrm{IH}$ & Ukraine & $72.7 \mathrm{k}$ & $15.3 \mathrm{i}-\mathrm{m}$ & $42.61-n$ & MS \\
\hline Elegant & $\mathrm{B}$ & $\mathrm{IH}$ & Russia & $82.0 \mathrm{~lm}$ & $12.0 \mathrm{~g}-\mathrm{i}$ & $45.81-0$ & MS \\
\hline Nadiezhda AZOS & $\mathrm{N}$ & $\mathrm{IH}$ & Russia & $79.3 \mathrm{~lm}$ & $15.9 \mathrm{k}-\mathrm{m}$ & $46.9 \mathrm{~m}-\mathrm{o}$ & MS \\
\hline Sfinks & $\mathrm{N}$ & $\mathrm{IH}$ & Ukraine & 78.71 & $16.6 \mathrm{k}-\mathrm{m}$ & $47.0 \mathrm{~m}-\mathrm{o}$ & MS \\
\hline Talisman & $\mathrm{B}$ & $\mathrm{IH}$ & Russia & $79.4 \mathrm{~lm}$ & $26.7 \mathrm{n}$ & $53.6 \mathrm{n}-\mathrm{p}$ & MS \\
\hline Galbena Nou & $\mathrm{B}$ & $\mathrm{IH}$ & Russia & $80.7 \mathrm{~lm}$ & $43.3 \mathrm{p}$ & $63.0 \mathrm{o}-\mathrm{q}$ & MS \\
\hline Danmarpa Polonia & $\mathrm{B}$ & $\operatorname{Vin}$ & Poland & $84.7 \mathrm{~m}$ & $46.6 \mathrm{p}$ & $67.2 \mathrm{pq}$ & MS \\
\hline Europleven & $\mathrm{B}$ & $\mathrm{IH}$ & Bulgaria & 95.7 o & 30.6 no & $68.4 \mathrm{pq}$ & $\mathrm{MS}$ \\
\hline Favor & $\mathrm{R}$ & $\mathrm{IH}$ & Russia & $91.4 \mathrm{n}$ & $51.3 \mathrm{q}$ & $74.0 \mathrm{q}$ & MS \\
\hline Stout Sdl & $\mathrm{B}$ & $\mathrm{IH}$ & USA & $91.4 \mathrm{n}$ & $51.3 \mathrm{q}$ & $74.0 \mathrm{q}$ & MS \\
\hline Vienetchnij & $\mathrm{B}$ & $\mathrm{IH}$ & Ukraine & $99.6 \mathrm{p}$ & 30.0 no & $74.5 \mathrm{q}$ & MS \\
\hline Philipp & $\mathrm{N}$ & $\mathrm{IH}$ & Austria & $98.7 \mathrm{p}$ & $46.7 \mathrm{p}$ & $79.8 \mathrm{qr}$ & $\mathrm{S}$ \\
\hline Evita & $\mathrm{B}$ & $\mathrm{IH}$ & Austria & $99.8 \mathrm{p}$ & $68.0 \mathrm{r}$ & $89.9 \mathrm{r}$ & $\mathrm{S}$ \\
\hline
\end{tabular}

Means followed by the same letter do not differ significantly at $\mathrm{p}=0.05$.

* Colour of berry skin: B (blanc) - green-yellow; $\mathrm{R}$ - rose; $\mathrm{N}$ (noir) - blue-black

** Origin: Vin $-V$. vinifera; $\mathrm{IH}$ - interspecific hybrid from crossing $V$. vinifera and species from among $V$. riparia, $V$. rupestris, $V$. lincecumii, $V$. amurensis and $V$. labrusca

*** Class of frost tolerance, with percentage of frozen buds in brackets: VR - very resistant (below $1.9 \%$ ), R - resistant ( $2 \%$ to $24.9 \%$ ), MS - medium susceptible (25\% to $74.9 \%$ ), $\mathrm{S}$ - susceptible (75\% to $95.9 \%$ ) and VS - very susceptible (above $96 \%)$. 
to most tolerant $V$. vinifera cultivars, such as 'Riesling', 'Tauberschwarz' and 'Pinot Noir'. The $V$. vinifera cultivars 'Dunaj' and 'Cabernet Mitos' were the most susceptible to frost damage.

Hybrids crossbred with $V$. labrusca in the USA 'Bluebell' and seedless 'Mars', 'Somerset', 'Trollhaugen', 'Venus', 'Jupiter' and 'Marquis', in Poland - 'Izopan', in Estonia - 'Kivikrass', in Canada - 'Vanessa' and the Russian hybrid crossbred with $V$. amurensis - 'Siewiernij Rannij', were table grape cultivars observed to be most frost resistant. The $V$. vinifera cultivar that proved most resistant to frost damage was the chimera, 'Tressot Panache'. Among interspecific hybrids with large, firm berries, 'Zodiak' was relatively resistant; its frost tolerance was similar to the reference hybrid 'Muscat Bleu', and definitely higher that the other reference cultivar, 'Chasselas Dore', which belong to $V$. vinifera. Most interspecific hybrids with attractive table grape berries originating from the Ukraine, Russia, Bulgaria, Hungary and Austria, with the exception of 'Zoltaja Lora' and 'Pleven Muskatnyj', proved to be more susceptible to frost damage than 'Chasselas Dore'. Most susceptible were buds of 'Philipp' and 'Evita'. Damage to the buds of $85 \%$ to $90 \%$ was accompanied by damage to one-year-old wood. The trunks and basal part of one-year-old shoots were not damaged, as the vines were covered with straw.

Planting frost-resistant cultivars is essential for the successful expansion of grapevine cultivation in central, eastern and northern Europe, Canada and the central and northern parts of the USA (Plocher \& Parke, 2001). The results presented here for the most part confirm data available in the existing literature, textbooks and Internet databases. Some of the results, however, differ from the available data, which could be attributed to differences in growing areas, climatic conditions, training system, cultural practices and vine condition (Fennel, 2004).

The present results confirm the high frost tolerance of wine and table grape cultivars grown in Canada and the USA; they meet the needs of central states such as Arkansas, and the northern states such as Wisconsin and Minnesota. 'Mars', 'Marquis', 'Jupiter' and 'Vanessa' are a group of resistant cultivars which, under American conditions, withstand temperatures between $-23^{\circ} \mathrm{C}$ and $-26^{\circ} \mathrm{C}$. 'Marquette', 'St. Croix', 'Sabrevois', 'Frontenac', 'Kay Gray', 'St. Pepin', 'Bluebell', 'Trollhaugen' and 'Somerset' (HORT 3040, 2016), and 'Vandal Cliché' (Bell, 2018) can all withstand a temperature below $-26^{\circ} \mathrm{C}$. Our own study confirmed American data that indicated that, among seedless cultivars grown in Arkansas (USA), 'Venus', 'Mars' and 'Jupiter' are more frost resistant than 'Saturn' (Bourne et al., 1991; Clark \& Moore, 1999).

In the course of the present study, traditional wine cultivars were proven to be medium susceptible to frost ('Merlot') or resistant ('Veltliner Frührot'), the same as in Slovakia (Pospišilova, 1981). Only some cultivars originating from Russia and Ukraine were confirmed to be very or relatively frost resistant under Polish conditions - as was declared in the respective countries of origin. 'Siewiernij Rannij' (from $-23^{\circ} \mathrm{C}$ to $-26^{\circ} \mathrm{C}$ ), 'Zodiak' (from $-23^{\circ} \mathrm{C}$ to $-26^{\circ} \mathrm{C}$ ), 'Zoltaja Lora' (from $-23^{\circ} \mathrm{C}$ to $-26^{\circ} \mathrm{C}$ ) and 'Chryzolit' $\left(-21^{\circ} \mathrm{C}\right)$ belonged to this group; the temperature ranges enclosed in the brackets indicate what temperatures the cultivars are supposed to withstand in Russia and Ukraine (Vinograd.info, 2019). Some of the cultivars, e.g. 'Favor' (from $-23^{\circ} \mathrm{C}$ to $\left.-26^{\circ} \mathrm{C}\right)$, 'Elegant' $\left(-25^{\circ} \mathrm{C}\right)$, 'Galbena Nou' $\left(-25^{\circ} \mathrm{C}\right)$, 'Vienetchnij' $\left(-23^{\circ} \mathrm{C}\right)$ and 'Sfinks' $\left(-21^{\circ} \mathrm{C}\right)$, were more susceptible to frost damage than declared in the abovementioned Russian source (the brackets enclose Russian data on the minimum temperatures that the buds of the cultivars can tolerate).

For the most part, our results confirm reference data from Germany, but they do differ in the case of several cultivars. As in Germany, 'Baron', 'Helios', 'Hibernal' and 'Tauberschwarz' were very resistant or resistant (Lott et al., 2010). However, 'Sirius' - described by Lott et al. (2010) as a cultivar characterised by frost resistance similar to that of 'Riesling', was proven by the research to be far more susceptible than the reference cultivar. 'Cabernet Mitos', assessed by the abovementioned German authors as equally resistant to frost damage as 'Pinot Noir', was also susceptible under Polish conditions. Lott et al. (2010) describe 'Solaris' and 'Regent' as resistant cultivars, but this and previous research conducted in Poland (Lisek, 2012) indicates that the buds of 'Regent' were more severely damaged by frost than the buds of 'Solaris'. Considerable differences were observed between the results obtained in our own research and the German results in the case of table grape cultivars. According to Lott et al. (2010), 'Romulus', 'Philipp', 'Evita', 'Sophie' and 'Katharina' are characterised by high frost resistance, whereas the results presented in this paper show that the buds of 'Evita' and 'Philipp' were strongly damaged by cold. Differences in defining varieties as cold resistant in Germany and Poland may result from the fact that, in Germany, frost resistance of $-16^{\circ} \mathrm{C}$ to $-18^{\circ} \mathrm{C}$ is sufficient. In Poland, however, temperature drops are greater than in Germany, which causes the conditions of field evaluation to be different and changes the criteria for recognising a variety as cold-resistant. The tendency for the buds of 'Evita' and 'Philipp' to freeze up is probably of genetic origin. The parental forms of both include varieties sensitive to frost: 'Evita' is F1 of 'Perlette', and 'Philipp' is F2 of 'Chaouch Rose' (Pospišilova, 1981; Nikov et al., 1983; Vitis International Variety Catalogue, 2019 ). 'Philipp' is characterised by late fruit ripening and high fertility, which may impede the maturation of the wood and the adaptation of buds in the winter under Polish conditions.

The research allowed us to assess, in terms of frost resistance, the cultivars that have not yet been documented in the methodical research available to the authors. Considering the mean results of the two winters, 'Allegro', 'Jutrzenka', 'Souvignier Gris' and 'Izopan' were very resistant. 'Villaris', 'Bolero', 'Cabernet Cantor', 'Felicia', 'Calandro', 'Muscaris', 'Turan' and 'Charlie' ('Antracit') were resistant, while 'Danmarpa Polonia' showed medium susceptibility.

The practical value of the present two-year study lies in the determination of the relationship between reference varieties and a large group of genotypes with cold resistance that is unknown in Poland. The reliability of these results is confirmed by the compliance of the data on reference varieties obtained in the present and historical studies carried out in the same collection and according to the same methodology 
(Lisek, 2009, 2012) and by other authors (Cindric \& Korac, 1990). In the present and historical research carried out in Skierniewice, different genotypes were assessed each time, but the reference cultivars of different origins and grape utility remained the same. These differences concern both the relationship between the cultivars and the results obtained within the cultivar, as the data on frost resistance may differ according to year of assessment, the physiological state of the plants, or the minimum temperatures and the time of their occurrence. Following the winter of 2009/2010, the buds of 'Pinot Noir' suffered more damage than the buds of 'Regent' (Lisek, 2012). The reverse relationship between these two cultivars was found after the winter of 2016/2017. Historical data show that the cold damage to the buds of the reference varieties after the winters of 2008/2009 and 2009/2010 was greater than following the winters of 2016/2017 and 2017/2018 (Table 4). The reason was a large drop in temperature found in the historical research, sometimes exceeding the values considered critical for the tested cultivars. The minimum temperature during the winter of 2008/2009 at the height of 2.0 metres as recorded on 2009-01-06 was $-23.0^{\circ} \mathrm{C}$. The minimum temperature of the next winter was $-28.1^{\circ} \mathrm{C}$, which was recorded on $2010-01$ 26. The pattern of cold resistance of the reference varieties after the winters of 2008/2009 and 2009/2010, in decreasing order, was as follows: 'Marechal Foch' $(\mathrm{R})>$ 'Muscat Bleu' (R) $>$ 'Riesling' $(\mathrm{MS})>$ 'Solaris' $(\mathrm{MS})>$ 'Regent' $(\mathrm{MS})>$ 'Chasselas Dore' (MS) > 'Pinot Noir' (S). After the winters of 2016/2017 and 2017/2018, the varieties were ranked in the following cold tolerance pattern: 'Marechal Foch' (VR) $>$ 'Riesling' (VR)' > 'Solaris' (VR) > 'Muscat Bleu' (R) $>$ 'Pinot Noir' $(R)>$ 'Chasselas Dore' $(R)>$ 'Regent' $(R)$. In both patterns, the first four cultivars, with greater frost resistance, and the next three, with lower frost resistance, were the same, although the order was slightly different. Also, Cindric and Korac (1990), who conducted a long-time study (six to nine years) in a cold chamber, with a temperature drop to $-21.0^{\circ} \mathrm{C}$, ranked the varieties according to decreasing frost resistance as: 'Riesling' > 'Pinot Noir' > 'Chasselas Dore', recognising all tolerant of cold damage. Both in the research by the abovementioned authors and in the present research conducted in Skierniewice, 'Merlot' buds were more severely damaged by frost than 'Riesling', 'Pinot Noir' and
'Chasselas Dore' buds. Although frost resistance is primarily determined by a genetic factor (Alleweldt et al., 1990), it is to some extent modified by the physiological state of plants (Cindric \& Korac, 1990). A heavy crop load (Evans, 2000) and fungal disease infestation (Plocher \& Parke, 2001) may reduce carbohydrate accumulation, weaken the vines and reduce cold hardiness. In the present study, the tendency to over-cropping was demonstrated in the interspecific hybrid 'Muscat Bleu', the inter-intraspecific hybrids 'Regent', 'Monarch' and 'Sirius' and, to a lesser extent, by 'Solaris'. In turn, among the varieties belonging to $V$. vinifera, 'Pinot Noir' was characterised by a greater tendency to overcropping and susceptibility to fungal diseases compared to 'Riesling'.

The results regarding frost resistance depend on the climate model of the growing area (occurrence of critical temperatures over time) and the genetic pattern of the varieties, which is responsible for the chemical composition of the grapevine tissues and the physiological and biochemical processes taking place in them (Ferguson et al., 2014; Nenko et al., 2018). However, in practical research on the frost resistance of varieties in a cold climate, the first step is to determine the critical temperature and compare the frost resistance of the tested and reference varieties, which was the aim of the present work. Our results were collected when the mid-winter (January to February) was frosty, which precluded the early termination of endodormancy and reduced the risk of damage to the buds due to late winter frosts.

Even a few decades ago, countries such as Poland, located around $50^{\circ} \mathrm{N}$ latitude, did not meet the conditions for commercial wine growing, which is recommended for regions where temperatures do not fall below $-20^{\circ} \mathrm{C}$ to $-22^{\circ} \mathrm{C}$, and they remain at this level for one to two days, not more often than once in five to six years (Nikov et al., 1983). Currently, climate changes are noticeable and are changing the regionalisation of grape growing (Rayne et al., 2011). As an example of these changes, we can mention unusually mild winters in central Poland (Skierniewice) in 2018/2019 and 2019/2020, when the temperature did not drop below $-12^{\circ} \mathrm{C}$. Thanks to the progress occurring in the breeding of varieties, valuable interspecific hybrids and inter-intraspecific (intra-interspecific) hybrids are available,

TABLE 4

Historical cold damage to reference wine grapes and table grapes following the winter of 2008/2009 and 2009/2010, Skierniewice.

\begin{tabular}{lcccc}
\hline & \multicolumn{3}{c}{ Frozen buds $(\%)$} & \\
\cline { 2 - 4 } Cultivar & $2008 / 2009$ & $2009 / 2010$ & Mean & Class of frost tolerance* (mean) \\
\hline Marechal Foch & $11.4 \mathrm{a}$ & $0.0 \mathrm{a}$ & $5.7 \mathrm{a}$ & $\mathrm{R}$ \\
Pinot Noir & $75.1 \mathrm{~g}$ & $85.0 \mathrm{~g}$ & $80.2 \mathrm{e}$ & $\mathrm{S}$ \\
Regent & $58.6 \mathrm{e}$ & $60.3 \mathrm{~d}$ & $59.4 \mathrm{~d}$ & $\mathrm{MS}$ \\
Riesling & $13.5 \mathrm{~b}$ & $60.5 \mathrm{e}$ & $37.0 \mathrm{c}$ & $\mathrm{MS}$ \\
Solaris & $50.5 \mathrm{~d}$ & $27.0 \mathrm{~b}$ & $38.6 \mathrm{c}$ & $\mathrm{MS}$ \\
Chasselas Dore & $60.1 \mathrm{f}$ & $62.1 \mathrm{f}$ & $61.3 \mathrm{~d}$ & $\mathrm{R}$ \\
Muscat Bleu & $14.4 \mathrm{c}$ & $29.0 \mathrm{c}$ & $21.7 \mathrm{~b}$ & $\mathrm{R}$ \\
\hline
\end{tabular}

Means followed by the same letter do not differ significantly at $\mathrm{p}=0.05$.

* Class of frost tolerance, with percentage of frozen buds in brackets: VR - very resistant (below 1.9\%), R - resistant (2\% to $24.9 \%)$, MS medium susceptible ( $25 \%$ to $74.9 \%)$, S - susceptible ( $75 \%$ to $95.9 \%$ ) and VS - very susceptible (above $96 \%$ ). 
for which critical temperatures range from $-20^{\circ} \mathrm{C}$ to $-25^{\circ} \mathrm{C}$. It is worth determining these varieties precisely in order to select the appropriate ones for cultivation and to minimise potential cold damage.

\section{CONCLUSIONS}

Frost resistance of the assessed grapevine cultivars within the $V$. vinifera species, as well as within the groups of interspecific and inter-intraspecific hybrids, varied extensively. Frost resistance of grapevine cultivars should be verified in region of cultivation.

\section{LITERATURE CITED}

Alleweldt, G., Spiegel-Roy, P. \& Reisch B.I., 1990. Grapes (Vitis). In: Moore, J.N. \& Ballington J.R. jr. (eds). Genetic resources of temperate fruit and nut crops. Acta Hortic. 290, 291-337.

Bell, R.A., 2018. Grape varieties in Canada - wines of Canada. Retrieved 15 July, 2019, from www.winesofcanada.com/grapes-quebec.html

Bourne, T.F., Moore, J.N. \& George, M.F., 1991. Primary bud hardiness of four genotypes of grapes in Arkansas. J. Am. Soc. Hortic. Sci. 116(5), $835-837$.

Cindric, P. \& Korac, N., 1990. Frost resistance of grapevine cultivars of different origin. Proc. 5th Intern. Symp. Grape Breeding, September 1989, St. Martin/Pfalz, FRG. Vitis (Special Issue) 29, 340-351.

Clark, J.R. \& Moore, J.N., 1999. 'Jupiter' seedless grape. HortScience 34(7), 1297-1299.

Evans, R.G., 2000. The art of protecting grapevines from low temperature injury. In: Rantz, J.M. (ed). Proc. ASEV 50th Anniv. Meeting, Seattle, Washington, June 2000. pp. $60-72$.

Fennel, A., 2004. Freezing tolerance and injury in grapevines. J. Crop Improv. 10(1-2), 201-235.

Ferguson, J., Moyer, M., Mills, L., Hoogenbom, G. \& Keller, M., 2014 Modeling dormant bud cold hardiness and budbreak in 23 Vitis genotypes reveals variation by region of origin. Am. J. Enol. Vitic. 65, 59-71.

Gustafsson, J.G. \& Mårtensson, A., 2005. Potential for extending Scandinavian wine cultivation. Acta Agric. Scand. B 55(2), 82-97.

HORT 3040, 2016. A review of cold climate grape cultivars. Iowa State University, Extension and Outreach. Retrieved 15 July, 2019, from https:// store.extension.iastate.edu/Product/A-Review-of-Cold-Climate-GrapeCultivars-pdf
Kostrikin, I.A., 1994. The breeding of complex resistance cultivars of grapevine in Russia. In: Troshin, L.P. (ed). Proc. VIth Intern. Symp. Grape Breeding, September 1994, Yalta, Crimea, Ukraine. pp. 89 - 90.

Lisek, J., 2009. Frost damage of buds on one-year-old shoots of wine and table grapevine cultivars in central Poland following the winter of 2008/2009. J. Fruit Ornam. Plant Res. 17(2), 149-161.

Lisek, J., 2012. Winter frost injury of buds on one-year-old grapevine shoots of Vitis vinifera cultivars and interspecific hybrids in Poland. Folia Hortic. 24(1), 97-103.

Lott, H., Pfaff, F. \& Prior, B., 2010. Taschenbuch der Rebsorten. 14 Auflage. Fachverlag Dr. Fraund GmbH, Mainz [in German].

Luby, J.J., 1991. Breeding cold-hardy fruit crops in Minnesota. HortScience 26(5), 505-512.

Nenko, I.A., Ilyina, I.A., Kiseleva, G.K. \& Yablonskay, E.K., 2018. The low-temperature stress tolerance of the grape varieties of ecological and geographical origin. J. Curr. Res. 10(11), 75740-75749.

Nikov, M., Jonev, S., Cholakov, T., Malenin, I., Todorov, I. \& Monov, I. 1983. Spravochnik po lozarstvo. Christo G. Danov Printing Co., Plovdiv [in Bulgarian].

Plocher, T.A. \& Parke, R.J., 2001. Northern winework. Eau Claire Printing Co., Minnesota.

Pospišilová, D., 1981. Ampelografia CSSR. Priroda, Bratislava [in Slovakian]

Rayne, S., Forest, K. \& Friesen, K.J., 2011. Projected climate change impacts on grape growing in the Okanagan Valley, British Columbia Canada. Nature Prec. https://doi.org/10.1038/npre.2009.3162.1

Reisch, B.I. \& Pratt, C., 1996. Grapes. In: Janick, J. \& Moore, J.N. (eds). Fruit breeding. Vol. II: Vine and small fruit crops. John Wiley \& Sons, Inc, New York. pp. 297 - 366

Theocharis, A., Hand, P., Pole, J., Cevik, V., Fisarakis, I. \& Henderson, J., 2010. Study of genetic diversity among inter-intraspecific hybrids and original grapevine varieties using AFLP molecular markers. Aust. J. Crop Sci. 4(1), 1-8.

Vinograd.info, 2019. Retrieved 15 July, 2019, from https:/vinograd.info/ sorta/stolovye/ [in Russian].

Vitis International Variety Catalogue, 2019. Retrieved 15 July, 2019, from www.vivc.de

Vool, E., Rätsep, R. \& Karp, K., 2015. Effect of genotype on grape quality parameters in cool climate condition. Acta Hort. 1082, 353-358. 